\section{P194 THE USE OF METHOTREXATE AS A STEROID SPARING AGENT IN SEVERE ASTHMA: A MULTI CENTRE RETROSPECTIVE ANALYSIS}

${ }^{1} \mathrm{~L}$ Holmes, ${ }^{2} \mathrm{~L}$ Afridi, ${ }^{2} \mathrm{~A}$ Dr Mansur, ${ }^{3} \mathrm{~S}$ Regan, ${ }^{3} \mathrm{~A}$ Menzies Gow, ${ }^{1} \mathrm{~S}$ Fowler, ${ }^{1} \mathrm{RM}$ Niven; ${ }^{1}$ University hospital of south manchester, Manchester, United Kingdom; ${ }^{2}$ Severe \& Brittle Asthma Unit Heart of England NHS Foundation Trust, Birmingham, United Kingdom; ${ }^{3}$ Royal Brompton \& Harefield NHS Foundation Trust, London, United Kingdom

\subsection{6/thoraxjn-2013-204457.346}

Background Methotrexate (MTX) immunosuppressive therapy is selectively used to assist with the reduction of the oral corticosteroid demand thereby decreasing the risk of potential side effects in individuals with steroid dependant asthma. Previous reported data has demonstrated similar significant reduction in corticosteroid load, asthma exacerbation and hospital admission rate. This study aims to compare similar variables collated from UK severe asthma specialist centres to further generate evidence for the continued use of MTX as an effective aid in the reduction of corticosteroid burden in a severe asthma cohort.

Methods A retrospective data collection was performed across two UK severe asthma centres with data from a third pending. Patients included had a confirmed diagnosis of severe asthma and had been treated with MTX for at least 12 months to allow sufficient analysis. Variables assessed included mean average daily corticosteroid dose, overall percentage reduction of corticosteroid, exacerbation frequency and acute admission events both twelve months prior to and post commencement of MTX therapy. Data collection was examined for each individual centre then combined and examined for consistency of results.

Results $N=29(21 / 8)$ Average daily corticosteroid dose was reduced by $6 \mathrm{mg}$ combined across both centres $[\mathrm{p}=0.004]$ $(6.4 \mathrm{mg} / 5 \mathrm{mg})$ a percentage of overall reduction of corticosteroid equated to $34.8 \%$ jointly across both centres and $39.3 \%$ and $30.0 \%$ individually. Exacerbation frequency decreased from 4.8 to 2.5 annually combined across both centres [ $p=0.003$ ] although conflict in result is identified as one centre reports a 1.0 increase in exacerbations compared to 3.4 decrease in the other centre. Combined admission decreased from 1.3 to 0.3 annually [ $\mathrm{p}=0.004] 1.1$ and 0.4 decrease in each individual centre.

Conclusion When monitored and supervised in the correctly chosen severe asthma patient, MTX can significantly reduce the demand for corticosteroids and consequently reduce steroid related side effects, admission and exacerbation this has been demonstrated across two specialist centres with correlating data.

\section{P195 PLUME CHARACTERISTICS OF FLUTICASONE PROPIONATE/FORMOTEROL PMDI COMPARED WITH FLUTICASONE PROPIONATE/SALMETEROL PMDI}

'B Johal, 'S Murphy, 'I Marshall; 'Mundipharma Research Limited, Cambridge, UK; ${ }^{2}$ Oxford Lasers Limited, Oxford, UK

\subsection{6/thoraxjn-2013-204457.347}

Background It has been suggested that aerosol inhaler characteristics such as fine particle size distribution, low plume velocity, and long duration of the aerosol cloud may assist coordination of inhalation with actuation, improve lung deposition, and reduce oropharyngeal deposition. This study compared 2 ICS/ LABA combination HFA pMDI inhalers: fluticasone propionate/ formoterol 125/5 $\mu \mathrm{g}$ (FP/FORM; flutiform $\left.{ }^{\circledR}\right)$ and fluticasone propionate/salmeterol 125/50 $\mu$ g (FP/SAL; Seretide ${ }^{\circledR}$ Evohaler $\left.^{\circledR}\right)$.
Methods Inhalers were operated according to their respective patient information leaflets, and were fired into still air. Plume data were recorded using an Oxford Lasers EnVision Pharma system with high speed CMOS camera and short-pulse laser light source. VidPIV 4.6 \& EnVision 1.1.5 software was used to analyse the data and assess velocity and other characteristics of the plume at set intervals from the actuator (up to $9.5 \mathrm{~cm}$ which is representative of the distance from mouth to throat).

Results FP/FORM pMDI had slower maximum velocity of plume than did FP/SAL at the distances measured (table). Furthermore, the duration of plume lasted approximately 50\% longer over the distance measured with FP/FORM than with FP/ SAL.

Conclusion FP/FORM has a slower and more prolonged plume compared with FP/SAL. This may help synchronise aerosol availability and inhalation and may lead to less oropharyngeal deposition and better lung deposition.

\begin{tabular}{lll} 
Abstract P195 Table 1. & & \\
\hline & FP/FORM & FP/SAL \\
Plume Duration over 95mm (ms) & 168.3 & 114.0 \\
Max velocity @ 30mm (m/s) & 20.3 & 26.5 \\
Max velocity @ 60mm $(\mathrm{m} / \mathrm{s})$ & 15.2 & 21.8 \\
Max velocity @ 95mm $(\mathrm{m} / \mathrm{s})$ & 10.1 & 15.6 \\
\hline
\end{tabular}

\section{Clinical ILD and OLD}

\section{P196 HEALTHCARE UTILISATION BY PATIENTS WITH IDIOPATHIC PULMONARY FIBROSIS; OBSERVATIONS FROM THE UK PIRFENIDONE NAMED PATIENT PROGRAMME}

${ }^{1} \mathrm{H}$ Parfrey, ${ }^{2} \mathrm{C}$ Leonard, ${ }^{3} \mathrm{MA}$ Gibbons, ${ }^{4} \mathrm{E}$ Armstrong, ${ }^{1} \mathrm{E}$ Harris, ${ }^{2} \mathrm{R}$ Frank, ${ }^{3} \mathrm{C}$ Sharp, ${ }^{5} \mathrm{~F}$ Percival; ${ }^{1}$ Papworth Hospital NHS Foundation Trust, Cambridge, United Kingdom; ${ }^{2}$ University Hospital of South Manchester NHS Foundation Trust, Manchester, United Kingdom; ${ }^{3}$ Royal Devon and Exeter NHS Foundation Trust, Exeter, United Kingdom; ${ }^{4}$ Royal Brompton and Harefield NHS Foundation Trust, London, United Kingdom; ${ }^{5} \mathrm{pH}$ Associates Ltd, Marlow, United Kingdom

\subsection{6/thoraxinl-2013-204457.348}

Introduction and Objectives There are limited data on the healthcare resource use that arises as a consequence of idiopathic pulmonary fibrosis (IPF). Navaratnam et $a l^{1}$ have recently reported rising hospital admissions due to IPF but utilisation of other health related services by individuals with IPF is unknown. As part of a real world assessment of the clinical experience of pirfenidone via the UK Named Patient Program data were collected to determine the burden placed on healthcare resources as a consequence of IPF.

Methods A multi-centre, retrospective, cohort review was undertaken across 4 NHS Trusts. Hospital resource use data were collected from the clinical records of individuals starting pirfenidone for IPF through the NPP before June 2012.

Results Data were available from 100 patients (76\% male) at baseline and 67 through to nine months from baseline. At baseline, the mean \pm S.D. age was $69.3 \pm 7.5$ years, mean \pm S.D. FVC $\%$ predicted was $70 \% \pm 19 \%$ and 62 patients were on oxygen therapy. In the first 6 months from baseline, 11 patients had 15 IPF-related hospitalisations of which 6 were for an acute exacerbation. One patient was hospitalised in the 6-9 months period. The mean \pm S.D. and median (IQR) hospital bed days in 
the first 6 months were $11.0 \pm 7.5$ and $11.0(6.0-13.5)$ days respectively for hospitalised patients and mean \pm S.D. $1.2 \pm 4.2$ days for all patients. One patient was admitted to the Intensive Care Unit, for 5 days. Eighteen patients had IPF-related Accident and Emergency department visits, 3 had an IPF-related day-case and 67 outpatient clinic visits, with a mean of $2.1 \pm 2.0$ outpatient clinic visits per patient in the first 6 months of the observation period.

Conclusion IPF is a terminal disease associated with significant morbidity and mortality. This is reflected in the high level of resource use and frequent accessing of health care services by this severely ill cohort of patients. Whether resource use has been positively impacted by the introduction of pirfenidone is unknown but merits prospective assessment.

\section{REFERENCE}

Navaratnam V, Fogarty AW, McKeever T, Hubbard RB. The Increasing Secondary Care Burden of Idiopathic Pulmonary Fibrosis Hospital Admission Trends in England From 1998 to 2010. CHEST 2013; 143(4):1078-1084

\section{P197 PHYSICAL INACTIVITY IN SARCOIDOSIS}

S Vasudevan, M Maddocks, S Chamberlain, A Spinou, C Wood, C Jolley, SS Birring; King's College London, London, United Kingdom

\subsection{6/thoraxinl-2013-204457.349}

Introduction Sarcoidosis is associated with dyspnoea, arthralgia, fatigue and poor health status. Little is known about physical activity in sarcoidosis. This study aimed to: 1) objectively measure physical activity in patients with pulmonary sarcoidosis; 2) investigate the relationship between physical activity and fatigue, exercise capacity, health status and lung function.

Methods 15 pulmonary sarcoidosis patients (mean age: 52.7 years; 4 males) and 14 healthy controls (mean age: 46.5 years; 4 males) were recruited. Physical activity was assessed objectively over one week, using a validated tri-axial accelerometer- Activ$\mathrm{Pal}^{\mathrm{TM}}$, and subjectively with the International Physical Activity Questionnaire (IPAQ). All participants also underwent pulmonary function tests, 6MWT and completed the Fatigue Assessment Scale (FAS), MRC Dyspnoea Scale and the King's Sarcoidosis QOL Questionnaire (KSQ).

Results Compared to controls, patients had lower mean \pm SD daily step count $(5624 \pm 1875$ steps vs. $10429 \pm 2942$ steps, p $<0.01$, figure 1) and time spent stepping $\left(1.18 \pm 0.35 \mathrm{hr}^{- \text {day }^{-1}}\right.$ vs. $\left.1.97 \pm 0.46 \mathrm{hr}^{-\mathrm{day}^{-1}} ; \mathrm{p}<0.01\right)$. There was also a trend to reduced bouts of activity in sarcoidosis patients compared with

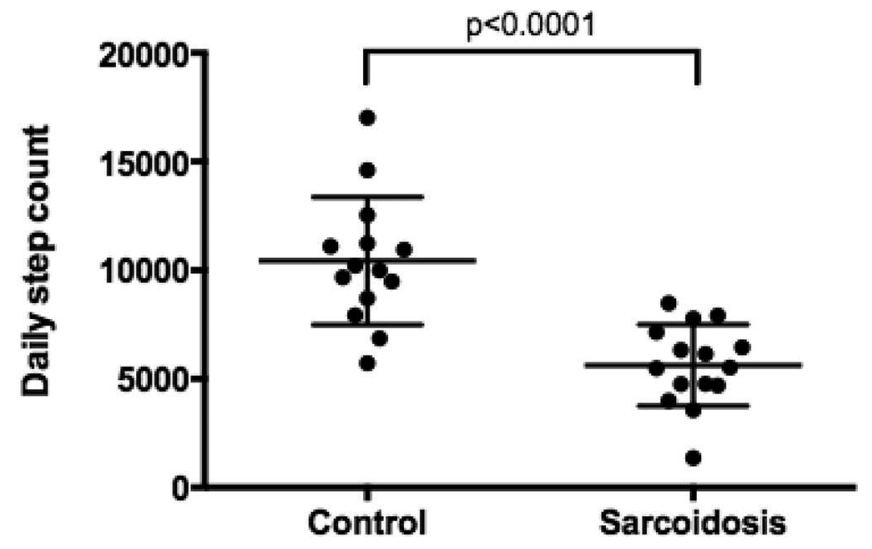

Abstract P197 Figure 1. Mean (SD) daily step count assessed with ActivPal. controls, (sit-stand transitions: $49 \pm 15$ vs. $61 \pm 20 ; \mathrm{p}=0.08$ ). Exercise capacity was significantly reduced in patients compared to controls (6MWT distance: $375 \pm 59$ m vs. $487 \pm 92 \mathrm{~m}$; p < $0.01)$. There was a significant association between daily step counts, and 6MWT distance, of patients $(\mathrm{r}=0.63, \mathrm{p}=0.01)$. Physical activity (overall) reported subjectively was not significantly different between groups (Overall IPAQ median score: 2153 vs. 3230 MET.min.week-1; p = 0.32). However, $87 \%$ of patients reported doing no vigorous physical activity compared to $50 \%$ in controls (vigorous activity IPAQ; $\mathrm{p}<0.01$ ). There were no significant correlations between daily step count and fatigue, dyspnoea, health status, lung function and self reported physical activity (IPAQ).

Conclusion Physical activity is significantly reduced in sarcoidosis compared to healthy subjects. Objective measures of physical activity assess a unique dimension of health in the patient's own environment that is not captured by existing clinical tools and should be further investigated.

\section{P198 ESTABLISHING THE PALLIATIVE AND SUPPORTIVE CARE NEEDS OF PATIENTS WITH IDIOPATHIC PULMONARY FIBROSIS AND NON SPECIFIC INTERSTITIAL PNEUMONIA}

${ }^{1} \mathrm{~J}$ Wall, ${ }^{2} \mathrm{~V}$ Crosby, ${ }^{2} \mathrm{~A}$ Hussain, ${ }^{2} \mathrm{~A}$ Wilcock, ${ }^{1} \mathrm{G}$ Saini, ${ }^{1} \mathrm{R}$ Braybrooke, ${ }^{1} \mathrm{G}$ Jenkins; ${ }^{1}$ Nottingham Respiratory Research Unit, University of Nottingham, Nottingham, England; ${ }^{2}$ Nottingham University Teaching Hospitals NHS Trust, Nottingham, England

\subsection{6/thoraxjnl-2013-204457.350}

Background Idiopathic Pulmonary Fibrosis (IPF) and Non Specific Interstitial Pneumonia (NSIP) are Idiopathic Interstitial Lung Diseases (ILD) without a known cure. IPF is progressive and fatal. Management of Idiopathic ILD must address patients' palliative and supportive care needs. The Sheffield Profile for Assessment and Referral to Care (SPARCC) questionnaire is a health needs assessment tool covering common biological, psychological and social needs. Local use of SPARC led to service refinements for thoracic cancer patients.

We now explore SPARC in patients with Idiopathic ILD to identify patients' perceptions of disease burden.

Methods Patients within 18 months of an Idiopathic ILD diagnosis, identified through specialist clinics, were invited to complete a SPARC questionnaire as part of an ongoing multicentre clinical study. Patients were excluded if concerns existed regarding ability to consent.

SPARC questionnaires included 45 questions: four (concerning personal, communication and information issues) required "yes/ no" responses. 41 questions related to symptoms and issues which were rated by patients as to the degree of distress they'd caused in the preceding month. Ratings were 0 "not at all", 1 "a little bit", 2 "quite a bit" and 3 "very much".

Responses were analysed using descriptive statistics. To highlight the most troubling issues, the percentage of patients reporting either "very much" or "quite a bit" of distress was identified for each question.

Results 97 patients with Idiopathic ILD (77 male) with median age 69 (range 47-86) were included. 79 (81\%) had IPF, 18 (19\%) NSIP. 38 (39\%) had severe lung function defects (TLCO $<40 \%)$.

Table 1 shows the 20 most common issues reported to cause "quite a lot" or "very much" distress.

Conclusions and implications Symptoms causing highest levels of distress in the Idiopathic ILD group reflect the commonly observed 'fibrotic triad' of dyspnoea, fatigue and cough. 